\title{
Squeezing Properties of Measurement Phase Operator in Superposed Squeezed Coherent States
}

\author{
Yongxin Zhan \\ Department of physics of SiChuan Agriculture university, Si Chuan, Ya An, 625014, China \\ Tel: 86-835-288-5166 E-mail: zyx073515@163.com
}

\begin{abstract}
The squeezing properties in the superposition of coherent States and squeezed state are investigated by means of the measurement phase operator introduced by Barnett and S M and Pagg D T.
\end{abstract}

Keywords: A new kind of odd and even coherent states, Measurement operator, Squeezing

\section{Introduction}

Recently, the discussion on phase of quantum optical field has given rise to a great many interest. It is known that in the quantum optical, the amplitude of field is directly proportional to square root of optic-quantum number operator and its phase is described by Susskind-Glogower phase operator $\exp ( \pm i \varphi)$, however Susskind-Glogower phase operator are possessed of unitary trait, hence hermit operator isn't constructed by it. Though two hermit operator below

$$
\begin{aligned}
& \cos \varphi=\frac{1}{2}[\exp (i \varphi)+\exp (-i \varphi)] \\
& \sin \varphi=\frac{1}{2}[\exp (i \varphi)-\exp (-i \varphi)]
\end{aligned}
$$

are set, they have classical property because $\left\langle\cos ^{2} \varphi\right\rangle+\left\langle\sin ^{2} \varphi\right\rangle \neq 1$. On purpose to overcome the difficulty, unitary exponential phase operator and measurement phase operator in optical field have defined by Pegg and Branett, and then the phase essence of optical field is studied in progress(PEGG D T, BARNETT S M. 1989)(BARNETT S M, PEGG D T. 1986). In some laboratories, the measurement phase operator usually corresponds to the measurement of phase, therefore the measurement phase operator has raised extensive concerns. Some classical properties are researched in detail for squeezing states, quasi-optical coherent states, squeezing optical number states, odd and even coherent states, Schrödinger cat states and so on.

In the quantum optics, coherent state and squeezed state are two very important states in the quantum optics(LYNCH R. 1987)(WALL D F. 1983). Coherent state is introduced by Schrödinger at first as most classical state in pure quantum state, which is eigenstate of annihilation operator $\hat{a}$, whose two variances of orthogonal amplitude of vibration are equal and they satisfy with minimal uncertainty relation. Squeezed state is squeezing transition vacuum state, whose one orthogonal amplitude variance is less than coherent state, however the other is more than coherent state. Squeezed state has very important application in interference measurement in high degree of accuracy, photo-communication as well as detection of gravitation wave and microwave signal(LOUDON R, KNIGHT P L. 1987)(WALL D F. 1987). According to superposition principle, a great many of new quantum states are constructed through superposition of arbitrary state and many favorable works have done. But these superposition states are usually composed of the same sort state, such as superposition of odd-even coherent state or squeezed odd-even coherent state. Some of their non-classical properties on measurement operator have discussed in detail. Recently the new superposition state composed of coherent state and squeezed state is studied and its quantum effect is obtained. In the paper, the squeezed properties of the superposition state on measurement operator are researched by mean of method in the paper(LOUDON R, KNIGHT P L. 1987).

\section{The superposition of squeezed coherent states}

Superposed coherent states is defined

$$
\mid \beta, \delta>=N\left[\left|\beta>+e^{i \delta}\right|-\beta>\right],
$$

where $\mid \beta>$ and $\mid-\beta>$ are the coherent states, $N=\left\{2\left[1+\cos \delta \exp \left(-2|\beta|^{2}\right)\right\}^{-1 / 2}\right.$ is normal parameter. When $\delta=0, \pi$ there are below two equations

$$
|\beta, 0>=N[|\beta>+|-\beta>],| \beta, \pi>=N[|\beta>-|-\beta>]
$$


They are even coherent and odd coherent states respectively.

After squeezing operator $S(\xi)=\exp \left[\frac{1}{2}\left(\xi^{*} a^{2}-\xi a^{+2}\right)\right]$ acts on coherent $\mid \beta>$, it becomes squeezed coherent states $\mid \beta>_{g}$, and there is

$\left|\beta>_{g}=S(\xi)\right| \beta>=\sum_{n=0}^{\infty}(u n !)^{-1 / 2}\left(\frac{v}{2 u}\right)^{n / 2} H_{n}\left(\frac{\beta}{\sqrt{2 u v}}\right) \exp \left(-\frac{|\beta|^{2}}{2}+\frac{v^{*} \beta^{2}}{2 u}\right)$

where $\beta=|\beta| e^{i \phi}, \xi=r e^{i \theta}, u=\cosh r, v=e^{i \theta} \sinh r,|\beta|$ and $\phi$ denotes the intensity and phase of coherent $\mid \beta>, r$ is squeezing factor, $\theta$ is squeezing angle, $H_{n}(z)$ is hermite polynomial.

By the same method, squeezing operator $S(\xi)$ act on (3), and then obtain squeezed coherent $\mid \beta, \delta>_{g}$

$$
\mid \beta, \delta>_{g}=N\left[\left|\beta>_{g}+e^{i \delta}\right|-\beta>_{g}\right]
$$

When $\delta=0$ and $\pi$,(6) become squeezed even odd coherent states

$$
\begin{aligned}
& \mid \beta, 0>_{g}=N\left[\left|\beta>_{g}+\right|-\beta>_{g}\right] \\
& \mid \beta, \pi>_{g}=N\left[\left|\beta>_{g}-\right|-\beta>_{g}\right]
\end{aligned}
$$

The operator $\hat{b}$ is defined below

$$
\hat{b}=u \hat{a}+v \hat{a}^{+}, \hat{b}^{+}=u^{*} \hat{a}^{+}+v^{*} \hat{a},
$$

where $\hat{a}$ and $\hat{a}^{+}$are Bose annihilation and creating operator, operator $\hat{b}$ and $\hat{b}^{+}$have the same as commutation relation $\hat{a}$ and $\hat{a}^{+}$,i.e. $\left[\hat{a}, \hat{a}^{+}\right]=\left[\hat{b}, \hat{b}^{+}\right]=1$, and

$\hat{b}\left|\beta, \delta>_{g}=\beta\right| \beta, \delta>_{g}$.

Above expression illustrates that $\mid \beta, \delta>_{g}$ is eigenstates of $\hat{b}$. For next requirement, sign is introduced

$$
\mid \beta, \delta>_{g, t}=N\left[\left|\beta>_{g}-e^{i \delta}\right|-\beta>_{g}\right]
$$

the equations are derived ( $m$ is integral number) from (6), (10) and (11)

$$
\begin{aligned}
\hat{b}^{2 m+1} \mid \beta, \delta>_{g} & =\beta^{2 m+1}\left|\beta, \delta>_{g, t}, \quad \hat{b}^{2 m}\right| \beta, \delta>_{g}=\beta^{2 m} \mid \beta, \delta>_{g} . \\
& <\beta, \delta \mid \beta, \delta>_{g}=1, \\
& <\beta, \delta \mid \beta, \delta>_{g, t}=-2 i N^{2} \sin \delta \exp \left(-2|\beta|^{2}\right), \\
& { }_{g, t}<\beta, \delta \mid \beta, \delta>_{g, t}=\frac{1-\cos \delta \exp \left(-2|\beta|^{2}\right)}{1+\cos \delta \exp \left(-2|\beta|^{2}\right)}=C
\end{aligned}
$$

\section{Orthogonal measurement phase operator}

Pegg and Barnnet have defined orthogonal measurement phase operator below

$$
\cos _{M} \varphi=\lambda X_{1}, \quad \sin _{M} \varphi=\lambda X_{2}
$$

where $X_{1}=\frac{1}{2}\left(\hat{a}+\hat{a}^{+}\right), \quad X_{2}=\frac{1}{2 i}\left(\hat{a}-\hat{a}^{+}\right) . \quad$ According to the non-classical requirement of 
$<\cos _{M}^{2} \varphi \mid \sin _{M}^{2} \varphi>=1 \lambda=(\bar{n}+1 / 2)^{-1 / 2}$, where $\bar{n}$ is average photon number in considered states in the paper, $\bar{n}=<\hat{a}^{+} \hat{a}>=<N>$.

By means of the relation of measurement phase operator and optical number operator, the important equations are got

$$
\begin{aligned}
& {\left[\cos _{M} \varphi, \sin _{M} \varphi\right]=\frac{i}{2} \lambda^{2},} \\
& {\left[\cos _{M} \varphi, N\right]=i \sin _{M} \varphi,} \\
& {\left[\sin _{M} \varphi, N\right]=-i \cos _{M} \varphi .}
\end{aligned}
$$

\section{Squeezing of measurement phase operator}

In quantum-optical field, when two operators $Y_{1}$ and $Y_{2}$ don't satisfy reciprocal relation, its accuracy of measurement is restricted by measurement indeterminacy principle below

$$
<\left(\Delta Y_{1}\right)^{2}><\left(\Delta Y_{2}\right)^{2}>>\frac{1}{4}\left|\left[Y_{1}, Y_{2}\right]\right|
$$

If the inequality is right

$$
<\left(\Delta Y_{1}\right)^{2}><\frac{1}{2}\left|\left[Y_{1}, Y_{2}\right]\right|, \quad(i=1,2)
$$

then there is squeezing effect in optical field component $Y_{i}$. With the view of description degree of squeezing, $S_{i}$ is defined

$$
S_{i}=<\left(\Delta Y_{1}\right)^{2}>-\frac{1}{2}\left|\left[Y_{1}, Y_{2}\right]\right|, \quad(i=1,2)
$$

When $S_{i}<0$, it indicates that there is squeezing in the $Y_{i}$.

From the expression (15), (16), the squeezing degrees of measurement phase operator are

$$
\begin{aligned}
S_{1}^{C S} & =<\left(\Delta \cos _{M} \varphi\right)^{2}>-\frac{1}{4}|\lambda|^{2}=\frac{\lambda^{2}}{4}<\hat{a}^{2}+\hat{a}^{+2}>-\frac{\lambda^{2}}{4}<\hat{a}+\hat{a}^{+}>^{2}+\frac{\lambda^{2}}{2}<\hat{a}^{+} \hat{a}> \\
& =\frac{\lambda^{2}}{4}\left[2<\hat{a}^{+} \hat{a}>+<\hat{a}^{2}+\hat{a}^{+2}>-<\hat{a}+\hat{a}^{+}>^{2}\right] \\
S_{2}^{C S} & =<\left(\Delta \sin { }_{M} \varphi\right)^{2}>-\frac{1}{4}|\lambda|^{2}=-\frac{\lambda^{2}}{4}<\hat{a}^{2}+\hat{a}^{+2}>+\frac{\lambda^{2}}{4}<\hat{a}-\hat{a}^{+}>^{2}+\frac{\lambda^{2}}{2}<\hat{a}^{+} \hat{a}> \\
& =\frac{\lambda^{2}}{4}\left[2<\hat{a}^{+} \hat{a}>+<\hat{a}-\hat{a}^{+}>^{2}-<\hat{a}^{2}+\hat{a}^{+2}>\right] \\
S^{C N} & =<\left(\Delta \cos _{M} \varphi\right)^{2}>-\frac{1}{2}\left|<\sin _{M} \varphi>\right| \\
& =\frac{\lambda^{2}}{4}<\hat{a}^{2}+\hat{a}^{+2}>-\frac{\lambda^{2}}{4}<\hat{a}+\hat{a}^{+}>^{2}-\frac{|\lambda|}{4}\left|<\hat{a}-\hat{a}^{+}>\right|+\frac{\lambda^{2}}{2}<\hat{a}^{+} \hat{a}>+\frac{\lambda^{2}}{4} \\
& =\frac{\lambda^{2}}{4}\left[2<\hat{a}^{+} \hat{a}>+1+<\hat{a}^{2}+\hat{a}^{+2}>-<\hat{a}+\hat{a}^{+}>^{2}\right]-\frac{|\lambda|}{4}\left|<\hat{a}-\hat{a}^{+}>\right|
\end{aligned}
$$




$$
\begin{aligned}
S^{S N} & =<\left(\Delta \sin _{M} \varphi\right)^{2}>-\frac{1}{2}\left|<\cos _{M} \varphi>\right| \\
& =-\frac{\lambda^{2}}{4}<\hat{a}^{2}+\hat{a}^{+2}>+\frac{\lambda^{2}}{4}<\hat{a}-\hat{a}^{+}>^{2}-\frac{|\lambda|}{4}\left|<\hat{a}+\hat{a}^{+}>\right|+\frac{\lambda^{2}}{2}<\hat{a}^{+} \hat{a}>+\frac{\lambda^{2}}{4} \\
& =\frac{\lambda^{2}}{4}\left[2<\hat{a}^{+} \hat{a}>+1-<\hat{a}^{2}+\hat{a}^{+2}>+<\hat{a}-\hat{a}^{+}>^{2}\right]-\frac{|\lambda|}{4}\left|<\hat{a}+\hat{a}^{+}>\right|
\end{aligned}
$$

In order to calculate above four values, $\hat{a}$ and $\hat{a}^{+}$are denoted by $\hat{b}$ and $\hat{b}^{+}$

$$
\hat{a}=u^{*} \hat{b}-v \hat{b}^{+}, \hat{a}^{+}=u \hat{b}^{+}-v^{*} \hat{b}
$$

$\hat{a}+\hat{a}^{+}=\left(u^{*}-v^{*}\right) \hat{b}+(u-v) \hat{b}^{+}$

$$
=\left(\cosh r-e^{-i \theta} \sinh r\right) \hat{b}+\left(\cosh r-e^{i \theta} \sinh r\right) \hat{b}^{+}
$$

$\hat{a}-\hat{a}^{+}=\left(u^{*}+v^{*}\right) \hat{b}-(u+v) \hat{b}^{+}$

$$
=\left(\cosh r+e^{-i \theta} \sinh r\right) \hat{b}-\left(\cosh r+e^{i \theta} \sinh r\right) \hat{b}^{+}
$$

$\hat{a}^{+} \hat{a}=\left(|u|^{2}+|v|^{2}\right) \hat{b}^{+} \hat{b}-u^{*} v^{*} \hat{b}^{2}-u v \hat{b}^{+2}+|v|^{2}$

$$
=\cosh 2 r \hat{b}^{+} \hat{b}-\frac{1}{2} e^{-i \theta} \sinh 2 r \hat{b}^{2}-\frac{1}{2} e^{i \theta} \sinh 2 r \hat{b}^{+2}+\sinh ^{2} r
$$

$$
\hat{a}^{2}=u^{* 2} \hat{b}^{2}+v^{2} \hat{b}^{+2}-2 u^{*} v \hat{b}^{+} \hat{b}-u^{*} v
$$$$
\hat{a}^{+2}=u^{2} \hat{b}^{+2}+v^{* 2} \hat{b}^{2}-2 u v^{*} \hat{b}^{+} \hat{b}-u v^{*}
$$$$
\hat{a}^{2}+\hat{a}^{+2}=\left(u^{* 2}+v^{* 2}\right) \hat{b}^{2}+\left(u^{2}+v^{2}\right) \hat{b}^{+2}-2\left(u^{*} v+u v^{*}\right) \hat{b}^{+} \hat{b}-\left(u^{*} v+u v^{*}\right)
$$$$
=\left(\cosh ^{2} r+e^{-2 i \theta} \sinh ^{2} r\right) \hat{b}^{2}+\left(\cosh ^{2} r+e^{2 i \theta} \sinh ^{2} r\right) \hat{b}^{+2}
$$$$
-2 \sinh 2 r \cos \theta \hat{b}^{+} \hat{b}-2 \sinh 2 r \cos \theta
$$

\section{The discussion of Squeezing effect of measurement phase operator}

Through calculating, the expressions below are obtained

$$
\begin{aligned}
& \hat{a}^{+} \hat{a}=\left(|u|^{2}+|v|^{2}\right) \hat{b}^{+} \hat{b}-u^{*} v^{*} \hat{b}^{2}-u v \hat{b}^{+2}+|v|^{2} \\
& =\cosh 2 r \hat{b}^{+} \hat{b}-\frac{1}{2} e^{-i \theta} \sinh 2 r \hat{b}^{2}-\frac{1}{2} e^{i \theta} \sinh 2 r \hat{b}^{+2}+\sinh ^{2} r \\
& \bar{n}=<\hat{a}^{+} \hat{a}>=_{g}<\beta, \delta\left|\left(\operatorname{ch} 2 r \hat{b}^{+} \hat{b}-\frac{1}{2} e^{-i \theta} \operatorname{sh} 2 r \hat{b}^{2}-\frac{1}{2} e^{i \theta} \operatorname{sh} 2 r \hat{b}^{+2}+s h^{2} r\right)\right| \beta, \delta>_{g} \\
& =|\beta|^{2} \operatorname{ch} 2 r_{g, t}<\beta, \delta\left|\beta, \delta>_{g, t}-\frac{1}{2} e^{-i \theta} \beta^{2} \operatorname{sh} 2 r_{g}<\beta, \delta\right| \beta, \delta>_{g} \\
& -\frac{1}{2} e^{i \theta} \beta^{* 2} \operatorname{sh} 2 r_{g}<\beta, \delta\left|\beta, \delta>_{g}+s^{2} r_{g}<\beta, \delta\right| \beta, \delta>_{g} \\
& =C|\beta|^{2} \operatorname{ch} 2 r-\frac{1}{2} e^{-i \theta} \beta^{2} \operatorname{sh} 2 r-\frac{1}{2} e^{i \theta} \beta^{* 2} \operatorname{sh} 2 r+\operatorname{sh}^{2} r \\
& =C|\beta|^{2} \operatorname{ch} 2 r-\frac{1}{2}|\beta|^{2} \operatorname{sh} 2 r\left(e^{-i \theta+i 2 \phi}+e^{i \theta-i 2 \phi}\right)+\operatorname{sh}^{2} r \\
& =C|\beta|^{2} \operatorname{ch} 2 r-|\beta|^{2} \operatorname{sh} 2 r \cos (\theta-2 \phi)+\operatorname{sh}^{2} r
\end{aligned}
$$




$$
\begin{aligned}
& \bar{n}=C|\beta|^{2} \cosh 2 r-|\beta|^{2} \sinh 2 r \cos (\theta-2 \phi)+s^{2} r \\
& \hat{a}+\hat{a}^{+}=\left(u^{*}-v^{*}\right) \hat{b}+(u-v) \hat{b}^{+} \\
& =\left(\operatorname{ch} r-e^{-i \theta} s h r\right) \hat{b}+\left(\operatorname{ch} r-e^{i \theta} s h r\right) \hat{b}^{+} \\
& <\hat{a}+\hat{a}^{+}>={ }_{g}<\beta, \delta\left|\left(\hat{a}+\hat{a}^{+}\right)\right| \beta, \delta>_{g} \\
& ={ }_{g}<\beta, \delta \mid\left[\left(\text { chr }-e^{-i \theta} \operatorname{sh} r\right) \hat{b}+\left(\text { chr }-e^{i \theta} \text { shr }\right) \hat{b}^{+}\right] \mid \beta, \delta>_{g} \\
& =\left(\operatorname{ch} r-e^{-i \theta} s h r\right)_{g}<\beta, \delta|\hat{b}| \beta, \delta>_{g}+\left(\operatorname{ch} r-e^{i \theta} \operatorname{shr}\right)_{g}<\beta, \delta\left|\hat{b}^{+}\right| \beta, \delta>_{g} \\
& =\beta\left(\operatorname{ch} r-e^{-i \theta} \text { shr }\right)_{g}<\beta, \delta\left|\beta, \delta>_{g, t}+\beta^{*}\left(\operatorname{ch} r-e^{i \theta} \operatorname{shr}\right)_{g, t}<\beta, \delta\right| \beta, \delta>_{g} \\
& =\beta\left(\operatorname{ch} r-e^{-i \theta} \operatorname{sh} r\right)\left(-2 i N^{2} \sin \delta \exp \left(-2|\beta|^{2}\right)\right)+\beta^{*}\left(\operatorname{ch} r-e^{i \theta} \operatorname{sh} r\right)\left(2 i N^{2} \sin \delta \exp \left(-2|\beta|^{2}\right)\right) \\
& =2 i N^{2}|\beta| c h r \sin \delta \exp \left(-2|\beta|^{2}\right)\left(e^{-i \phi}-e^{i \phi}\right)+2 i N^{2}|\beta| \operatorname{sh} r \sin \delta \exp \left(-2|\beta|^{2}\right)\left(e^{-i(\theta-\phi)}-e^{i(\theta-\phi)}\right) \\
& =-2 i N^{2}|\beta| \operatorname{ch} r \sin \delta \exp \left(-2|\beta|^{2}\right)\left(e^{i \phi}-e^{-i \phi}\right)-2 i N^{2}|\beta| \operatorname{sh} r \sin \delta \exp \left(-2|\beta|^{2}\right)\left(e^{i(\theta-\phi)}-e^{-i(\theta-\phi)}\right) \\
& =4 N^{2}|\beta| \operatorname{ch} r \sin \phi \sin \delta \exp \left(-2|\beta|^{2}\right)+4 N^{2}|\beta| \operatorname{sh} r \sin (\theta-\phi) \sin \delta \exp \left(-2|\beta|^{2}\right) \\
& =4 N^{2}|\beta| \sin \delta[\operatorname{ch} r \sin \phi+\operatorname{sh} r \sin (\theta-\phi)] \exp \left(-2|\beta|^{2}\right) \\
& <\hat{a}+\hat{a}^{+}>=4 N^{2}|\beta| \sin \delta[\cosh r \sin \phi+\sinh r \sin (\theta-\phi)] \exp \left(-2|\beta|^{2}\right) \\
& \hat{a}-\hat{a}^{+}=\left(u^{*}+v^{*}\right) \hat{b}-(u+v) \hat{b}^{+} \\
& =\left(\operatorname{ch} r+e^{-i \theta} \operatorname{sh} r\right) \hat{b}-\left(\operatorname{ch} r+e^{i \theta} \operatorname{sh} r\right) \hat{b}^{+} \\
& <\hat{a}-\hat{a}^{+}>={ }_{g}<\beta, \delta\left|\left(\hat{a}-\hat{a}^{+}\right)\right| \beta, \delta>_{g} \\
& ={ }_{g}<\beta, \delta\left|\left[\left(\operatorname{ch} r+e^{-i \theta} \operatorname{sh} r\right) \hat{b}-\left(\operatorname{ch} r+e^{i \theta} \operatorname{shr}\right) \hat{b}^{+}\right]\right| \beta, \delta>_{g} \\
& =\left(c h r+e^{-i \theta} s h r\right)_{g}<\beta, \delta|\hat{b}| \beta, \delta>_{g}-\left(c h r+e^{i \theta} s h r\right)_{g}<\beta, \delta\left|\hat{b}^{+}\right| \beta, \delta>_{g} \\
& =\beta\left(\operatorname{chr}+e^{-i \theta} \text { shr }\right)_{g}<\beta, \delta\left|\beta, \delta>_{g, t}-\beta^{*}\left(\operatorname{ch} r+e^{i \theta} \operatorname{sh} r\right)_{g, t}<\beta, \delta\right| \beta, \delta>_{g} \\
& =\beta\left(\operatorname{ch} r+e^{-i \theta} \operatorname{sh} r\right)\left(-2 i N^{2} \sin \delta \exp \left(-2|\beta|^{2}\right)\right)-\beta^{*}\left(\operatorname{ch} r+e^{i \theta} \operatorname{shr}\right)\left(2 i N^{2} \sin \delta \exp \left(-2|\beta|^{2}\right)\right) \\
& =-2 i N^{2}|\beta| \operatorname{ch} r \sin \delta \exp \left(-2|\beta|^{2}\right)\left(e^{i \phi}+e^{-i \phi}\right)-2 i N^{2}|\beta| \operatorname{shr} \sin \delta \exp \left(-2|\beta|^{2}\right)\left(e^{-i(\theta-\phi)}+e^{i(\theta-\phi)}\right) \\
& =-4 i N^{2}|\beta| \operatorname{chr} \cos \phi \sin \delta \exp \left(-2|\beta|^{2}\right)-4 i N^{2}|\beta| \operatorname{shr} \cos (\theta-\phi) \sin \delta \exp \left(-2|\beta|^{2}\right) \\
& =-4 i N^{2}|\beta| \sin \delta[\operatorname{chr} \cos \phi+\operatorname{shr} \cos (\theta-\phi)] \exp \left(-2|\beta|^{2}\right) \\
& <\hat{a}-\hat{a}^{+}>=-4 i N^{2}|\beta| \sin \delta[\cosh r \cos \phi+\sinh r \cos (\theta-\phi)] \exp \left(-2|\beta|^{2}\right) \\
& \hat{a}^{2}+\hat{a}^{+2}=\left(u^{* 2}+v^{* 2}\right) \hat{b}^{2}+\left(u^{2}+v^{2}\right) \hat{b}^{+2}-2\left(u^{*} v+u v^{*}\right) \hat{b}^{+} \hat{b}-\left(u^{*} v+u v^{*}\right) \\
& =\left(c h^{2} r+e^{-2 i \theta} s^{2} r\right) \hat{b}^{2}+\left(c h^{2} r+e^{2 i \theta} \operatorname{sh}^{2} r\right) \hat{b}^{+2}-2 \operatorname{sh} 2 r \cos \theta \hat{b}^{+} \hat{b}-s h 2 r \cos \theta \\
& <\hat{a}^{2}+\hat{a}^{+2}>={ }_{g}<\beta, \delta\left|\hat{a}^{2}+\hat{a}^{+2}\right| \beta, \delta>_{g} \\
& ={ }_{g}<\beta, \delta\left|\left[\left(c h^{2} r+e^{-2 i \theta} \operatorname{sh}^{2} r\right) \hat{b}^{2}+\left(\operatorname{ch}^{2} r+e^{2 i \theta} \operatorname{sh}^{2} r\right) \hat{b}^{+2}-2 \operatorname{sh} 2 r \cos \theta \hat{b}^{+} \hat{b}-\operatorname{sh} 2 r \cos \theta\right]\right| \beta, \delta>_{g} \\
& =\left(c h^{2} r+e^{-2 i \theta} s h^{2} r\right)_{g}<\beta, \delta\left|\hat{b}^{2}\right| \beta, \delta>_{g}+\left(c^{2} r+e^{2 i \theta} \operatorname{sh}^{2} r\right)_{g}<\beta, \delta\left|\hat{b}^{+2}\right| \beta, \delta>_{g} \\
& -2 \operatorname{sh} 2 r \cos \theta_{g}<\beta, \delta\left|\hat{b}^{+} \hat{b}\right| \beta, \delta>_{g}-\operatorname{sh} 2 r \cos \theta_{g}<\beta, \delta|| \beta, \delta>_{g} \\
& =\beta^{2}\left(\operatorname{ch}^{2} r+e^{-2 i \theta} \operatorname{sh}^{2} r\right)+\beta^{* 2}\left(\operatorname{ch}^{2} r+e^{2 i \theta} \operatorname{sh}^{2} r\right)-\operatorname{sh} 2 r \cos \theta\left(2 C|\beta|^{2}+1\right) \\
& =2|\beta|^{2} \operatorname{ch}^{2} r \cos 2 \phi+2|\beta|^{2} \operatorname{sh}^{2} r \cos (2 \theta-2 \phi)-\operatorname{sh} 2 r \cos \theta\left(2 C|\beta|^{2}+1\right)
\end{aligned}
$$


$<\hat{a}^{2}+\hat{a}^{+2}>=2|\beta|^{2} \cosh ^{2} r \cos 2 \phi+2|\beta|^{2} \sinh ^{2} r \cos (2 \theta-2 \phi)-\sinh 2 r \cos \theta\left(2 C|\beta|^{2}+1\right)$

Through calculating, the expressions below are obtained

$$
\begin{aligned}
S_{1}^{C S} & =<\left(\Delta \cos _{M} \varphi\right)^{2}>-\frac{1}{4}|\lambda|^{2}=\frac{\lambda^{2}}{4}<\hat{a}^{2}+\hat{a}^{+2}>-\frac{\lambda^{2}}{4}<\hat{a}+\hat{a}^{+}>^{2}+\frac{\lambda^{2}}{2}<\hat{a}^{+} \hat{a}> \\
& =\frac{\lambda^{2}}{4}\left[2<\hat{a}^{+} \hat{a}>+<\hat{a}^{2}+\hat{a}^{+2}>-<\hat{a}+\hat{a}^{+}>^{2}\right] \\
N & =\left\{2\left[1+\cos \delta \exp \left(-2|\beta|^{2}\right)\right\}^{-1 / 2} \quad{ }_{g, t}<\beta, \delta \mid \beta, \delta>_{g, t}=\frac{1-\cos \delta \exp \left(-2|\beta|^{2}\right)}{1+\cos \delta \exp \left(-2|\beta|^{2}\right)}=C\right.
\end{aligned}
$$

Put (19) into (17)

$$
\begin{aligned}
& S_{1}^{C S}=\frac{1}{4}\left[C|\beta|^{2} \operatorname{ch} 2 r-|\beta|^{2} \operatorname{sh} 2 r \cos (\theta-2 \phi)+s^{2} r+1 / 2\right]^{-1} \\
& \left\{2|\beta|^{2} \operatorname{ch}^{2} r \cos 2 \phi+2|\beta|^{2} \operatorname{sh}^{2} r \cos (2 \theta-2 \phi)-\operatorname{sh} 2 r \cos \theta\left(2 C|\beta|^{2}+1\right)\right.
\end{aligned}
$$$$
-\left[4 N^{2}|\beta| \sin \delta(\operatorname{ch} r \sin \phi+\operatorname{sh} r \sin (\theta-\phi)) \exp \left(-2|\beta|^{2}\right)\right]^{2}
$$$$
\left.+2\left(C|\beta|^{2} \operatorname{ch} 2 r-|\beta|^{2} \operatorname{sh} 2 r \cos (\theta-2 \phi)+s^{2} r\right)\right\}
$$$$
S_{1}^{C S}=\frac{1}{4}\left[C|\beta|^{2} \operatorname{ch} 2 r-|\beta|^{2} \operatorname{sh} 2 r \cos (\theta-2 \phi)+\operatorname{sh}^{2} r+1 / 2\right]^{-1}
$$$$
\left\{2|\beta|^{2} \operatorname{ch}^{2} r \cos 2 \phi+2|\beta|^{2} \operatorname{sh}^{2} r \cos (2 \theta-2 \phi)-\operatorname{sh} 2 r \cos \theta\left(2 C|\beta|^{2}+1\right)\right.
$$

$-\left[4 N^{2}|\beta| \sin \delta(\operatorname{chr} \sin \phi+\operatorname{shr} \sin (\theta-\phi)) \exp \left(-2|\beta|^{2}\right)\right]^{2}$

$$
\left.+2\left(C|\beta|^{2} \operatorname{ch} 2 r-|\beta|^{2} \operatorname{sh} 2 r \cos (\theta-2 \phi)+s^{2} r\right)\right\}
$$$$
S_{1}^{C S}=\frac{1}{4}\left[C|\beta|^{2} \operatorname{ch} 2 r-|\beta|^{2} \operatorname{sh} 2 r \cos (\theta-2 \phi)+\operatorname{sh}^{2} r+1 / 2\right]^{-1}
$$

$\left\{2|\beta|^{2}\left[\operatorname{ch}^{2} r \cos 2 \phi+\operatorname{sh}^{2} r \cos (2 \theta-2 \phi)-\operatorname{sh} 2 r \cos (\theta-2 \phi)\right]\right.$

$+\left(2 \operatorname{sh}^{2} r-\operatorname{sh} 2 r \cos \theta\right)+2 C|\beta|^{2}[\operatorname{ch} 2 r-\operatorname{sh} 2 r \cos \theta]$

$\left.-\left[4 N^{2} \exp \left(-4|\beta|^{2}\right)\right] 4 N^{2}|\beta|^{2} \sin ^{2} \delta[\operatorname{chr} \sin \phi+\operatorname{sh} r \sin (\theta-\phi)]^{2}\right\}$

$S_{1}^{C S}=\frac{1}{4}\left[C|\beta|^{2} \operatorname{ch} 2 r-|\beta|^{2} \operatorname{sh} 2 r \cos (\theta-2 \phi)+\operatorname{sh}^{2} r+1 / 2\right]^{-1}$

$\left\{2|\beta|^{2} \operatorname{ch}^{2} r \cos 2 \phi+2|\beta|^{2} \operatorname{sh}^{2} r \cos (2 \theta-2 \phi)-\operatorname{sh} 2 r \cos \theta\left(2 C|\beta|^{2}+1\right)\right.$

$-\left[4 N^{2}|\beta| \sin \delta(\operatorname{ch} r \sin \phi+s h r \sin (\theta-\phi)) \exp \left(-2|\beta|^{2}\right)\right]^{2}$

$$
\left.+2\left(C|\beta|^{2} \operatorname{ch} 2 r-|\beta|^{2} \operatorname{sh} 2 r \cos (\theta-2 \phi)+s^{2} r\right)\right\}
$$

$S_{1}^{C S}=\frac{1}{4}\left[C|\beta|^{2} \operatorname{ch} 2 r-|\beta|^{2} \operatorname{sh} 2 r \cos (\theta-2 \phi)+\operatorname{sh}^{2} r+1 / 2\right]^{-1}$

$\left\{2|\beta|^{2}\left[\operatorname{ch}^{2} r \cos 2 \phi+\operatorname{sh}^{2} r \cos (2 \theta-2 \phi)-\operatorname{sh} 2 r \cos (\theta-2 \phi)\right]\right.$

$+\left(2 \operatorname{sh}^{2} r-\operatorname{sh} 2 r \cos \theta\right)+2 C|\beta|^{2}[\operatorname{ch} 2 r-\operatorname{sh} 2 r \cos \theta]$

$\left.-\left[4 N^{2} \exp \left(-4|\beta|^{2}\right)\right] 4 N^{2}|\beta|^{2} \sin ^{2} \delta[\operatorname{chr} \sin \phi+\operatorname{sh} r \sin (\theta-\phi)]^{2}\right\}$ 
By means of numerical calculation technique, some of figures which indicate squeezing degree $S_{i}^{C S}(i=1,2)$, $S^{C N}$ and $S^{S N}$ varying with $\beta, r, \delta, \phi$ and $\theta$ are drawn. In these figures, Solid line denotes squeezing degree $S_{1}^{C S}$ and $S^{C N}$ curve varying with $\beta, r, \delta, \phi$ and $\theta$, dot line denotes $S_{2}^{C S}$ and $S^{S N}$ curve varying with $\beta, r, \delta, \phi$ and $\theta$. If $S_{i}^{C S}(i=1,2)$ is negative, it indicates that there is a kind of squeezing properties of $C S$. If $S^{C N}$ or $S^{S N}$ is negative, it indicates that there is a kind of squeezing properties of $C N$ or $S N$.

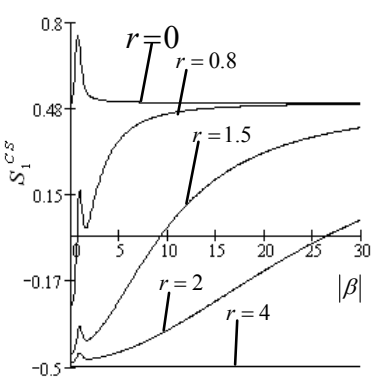

(a) $\delta=0$

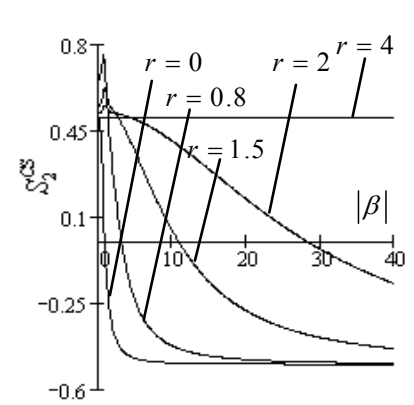

(e) $\delta=0$

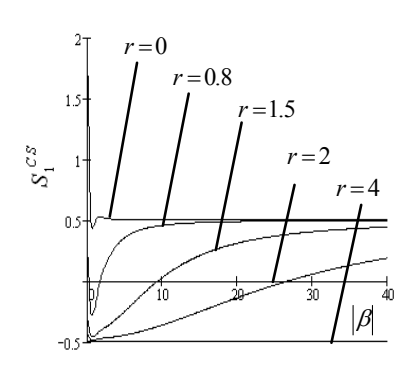

(b) $\delta=\pi$

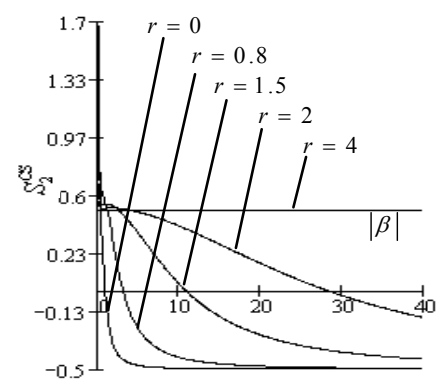

(f) $\delta=\pi$

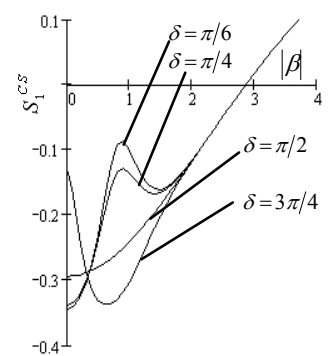

(d) $r=1$

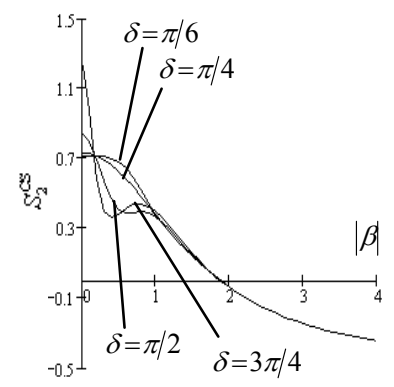

(g) $r=1$

Figure 1. The curve of degree of squeeze $S_{1}^{C S}$ and $S_{2}^{C S}$ varying with $\beta, r$ and $\delta$ at $\phi=\theta=0$

In fig (1), there are squeeze in squeeze odd-even coherent from fig (a), (b), (e) and (f) under different $r$ at $\phi=\theta=0$.

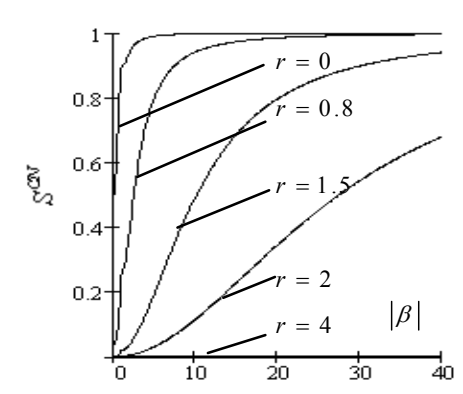

(a) $\delta=0, \pi$

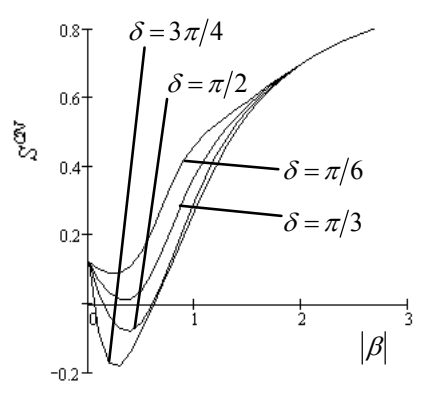

(b) $r=0.5$

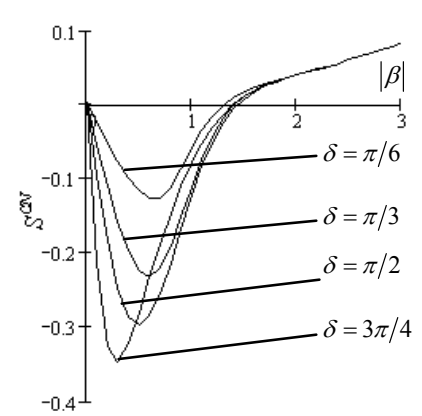

(c) $\delta=1.5$

Figure 2. The curve of degree of squeeze $S^{C N}$ varying with $\beta, r$ and $\delta$ at $\phi=\theta=0$

In the fig 2, fig (a) indicates that there are not $\mathrm{CN}$ squeeze in the squeezed odd-event coherent under different $r$ 
at $\phi=\theta=0$. Fig (b) and (c) indicate there are $\mathrm{CN}$ squeeze under different $\delta$, and the length of squeeze interval rise when $r$ increase. There is not $\mathrm{SN}$ squeeze.

\section{Conclusion}

In the paper, the squeeze properties of measurement phase operator are investigated in Superposition of Coherent State and Squeezed state.

\section{References}

BARNETT S M, PEGG D T. (1986). Phase in quantum optics. Phys (A) Math Gen, 1986, 19: 3849.

LOUDON R, KNIGHT P L. (1987). Squeezed light. J Mod Opt, 1987, 34(6-7): 709-759.

LYNCH R. (1987). Phase fluctuations in a squeezed state using measured phase operators. J Opt Soc Am (B), 1987, 4: 1723.

NATH R, KUMER P. (1991). Quasi-photon phase states. J Mod Opt, 1991, 38: 263.

PEGG D T, BARNETT S M. (1989). Phase properties of single-mode electromagnetic field. Phys Rev A, 1989, a39: 1665 .

TU HSITEH, GONG CHANGDE. (1993). Properties of the measured phase operators in squeezed number states. J Mod Opt, 1993, 40: 57.

WALL D F. (1983). Squeezed States of Light. Nature, 1983, 306(10): 141-146. 